\title{
Alcohol consumption pattern and risk of Barrett's oesophagus and erosive oesophagitis: an Italian case-control study
}

\author{
Rosa A. Filiberti ${ }^{1 *}$, Vincenzo Fontana ${ }^{1}$, Antonella De Ceglie ${ }^{2}$, Sabrina Blanchi ${ }^{2}$, Enzo Grossi ${ }^{3}$, \\ Domenico Della Casa ${ }^{4}$, Teresa Lacchin ${ }^{5}$, Marina De Matthaeis ${ }^{6}$, Orazio Ignomirelli ${ }^{7}$, Roberta Cappiello ${ }^{8}$, \\ Alessandra Rosa ${ }^{1}$, Monica Foti ${ }^{9}$, Francesco Laterza ${ }^{10}$, Vittorio D'Onofrio ${ }^{11}$, Gaetano Iaquinto ${ }^{11}$ and \\ Massimo Conio ${ }^{2}$ \\ ${ }^{1}$ Clinical Epidemiology, IRCCS AOU San Martino-IST, Largo R Benzi 10, 16132 Genova, Italy \\ ${ }^{2}$ Gastroenterology, General Hospital, Via G Borea 56, 18038 Sanremo, Imperia, Italy \\ ${ }^{3}$ Medical Department, Bracco Spa, Via E Folli 50, 20134 Milan, Italy \\ ${ }^{4}$ Digestive Endoscopic Surgery, Spedali Civili di Brescia, Piazzale Spedali Civili 1, 25123 Brescia, Italy \\ ${ }^{5}$ Endoscopy, Policlinico San Giorgio, Via Gemelli 10, 33170 Pordenone, Italy \\ ${ }^{6}$ Gastroenterology and Digestive Endoscopy, Ospedale di Lavagna, ASL 4 Chiavarese, Via Don Bobbio 25, \\ 16033 Lavagna, Italy \\ ${ }^{7}$ Endoscopy, IIRCCS, Centro di Riferimento Oncologico di Basilicata, Via Padre Pio 1, 85028 Rionero in Vulture, Potenza, Italy \\ ${ }^{8}$ Gastroenterology, S. Maria degli Angeli Hospital, Via Piave 54, 33170 Pordenone, Italy \\ ${ }^{9}$ Gastroenterology, LARC Private Clinic, Cso Venezia 10, 10155 Torino, Italy \\ ${ }^{10}$ Department of Internal Medicine, Unit of Endoscopy and Gastroenterology, University Hospital SS.Annunziata, \\ G.D’Annunzio University, Via dei Vestini, 66100 Chieti, Italy \\ ${ }^{11}$ Gastroenterology and Digestive Endoscopy, S. G. Moscati Hospital, Via San Giuseppe Moscati, 83100 Avellino, Italy \\ (Submitted 20 June 2016 - Final revision received 10 February 2017 - Accepted 27 March 2017 - First published online 8 May 2017)
}

\section{Abstract}

Knowledge about the association between alcohol and Barrett's oesophagus and reflux oesophagitis is conflicting. In this case-control study we evaluated the role of specific alcoholic beverages (red and white wine, beer and liquors) in 339 Barrett's oesophagus and 462 oesophagitis patients compared with 619 endoscopic controls with other disorders, recruited in twelve Italian endoscopic units. Data on alcohol and other individual characteristics were obtained from structured questionnaires. No clear, monotonic significant dose-response relationship was pointed out for red wine. However, a generalised U-shaped trend of Barrett's oesophagus/oesophagitis risk due to red wine consumption particularly among current drinkers was found. Similar results were also found for white wine. Liquor/spirit consumption seemed to bring about a 1.14-2.30 risk excess, although statistically non-significant, for current Barrett's oesophagus/oesophagitis drinkers. Statistically significant decreasing dose-response relationships were found in Barrett's oesophagus for frequency and duration of beer consumption. Similar, but less clear downward tendencies were also found for oesophagitis patients. In conclusion, although often not statistically significant, our data suggested a reduced risk of Barrett's oesophagus and oesophagitis with a low/moderate intake of wine and beer consumption. A non-significant increased risk of Barrett's oesophagus/ oesophagitis was observed with a higher intake of any type of heavy alcohol consumption, but no conclusion can be drawn owing to the high number of non-spirit drinkers and to the small number of drinkers at higher alcohol intake levels.

Key words: Barrett's oesophagus: Oesophagitis: Alcohol: Risk factors: Epidemiology: Gastro-oesophageal reflux disease

Barrett's oesophagus (BE) is a metaplastic transformation from the normal squamous mucosa of the oesophagus to a columnar lining and it is a known precursor for oesophageal adenocarcinoma $(\mathrm{EAC})^{(1,2)}$. Erosive oesophagitis (E) is not considered a precancerous lesion, but, like $\mathrm{BE}$, is associated with gastro-oesophageal reflux disease (GERD), a spectrum of hiatal hernia, gastro-oesophageal reflux and symptoms like heartburn and regurgitation ${ }^{(3)}$. Nevertheless, only a small fraction of patients with typical GERD symptoms have endoscopic evidence of $\mathrm{BE}$ or $\mathrm{E}^{(3,4)}$, although these disorders have also, in common, other potentially modifiable

Abbreviations: BE, Barrett's oesophagus; EAC, oesophageal adenocarcinoma; E, oesophagitis; GERD, gastro-oesophageal reflux disease; C, control; MLR, multinomial logistic regression; TLT, test for linear trend.

* Corresponding author: R. A. Filiberti, email rosa.filiberti@hsanmartino.it 
risk factors such as cigarette smoking and overweight ${ }^{(2,3,5)}$. Alcohol, in particular heavy alcohol consumption, has been associated with increased risk of oesophageal squamous cancer, but knowledge about the association between alcohol and reflux $\mathrm{E}, \mathrm{BE}$ and $\mathrm{EAC}$ is conflicting ${ }^{(6)}$. So far, most evidence supports no association between alcohol intake and BE risk ${ }^{(7-9)}$. On the other hand, an inverse correlation between wine intake and $\mathrm{BE}$ risk has been suggested ${ }^{(10)}$. A recent meta-analysis of twenty observational studies found no overall association between alcohol consumption and $\mathrm{BE}$, whereas in a subgroup analysis an increased risk of $\mathrm{BE}$ was found for overall alcohol consumption in men $(+35 \%)$ and for liquor consumption $(16 \%)^{(11)}$.

In this study we aimed to evaluate the role of specific alcoholic beverages in $\mathrm{BE}$ and $\mathrm{E}$ occurrence, compared with a group of control (C) subjects undergoing upper endoscopy, but with no BE or E.

\section{Methods}

A multicentre case-control study was carried out in twelve endoscopic units situated in different Italian areas (five in northern, two in central and five in southern Italy). Three groups of patients who were willing to answer a questionnaire were consecutively selected from those referred for upper gastrointestinal endoscopy: BE patients, $\mathrm{E}$ patients and $\mathrm{C}$ subjects without $\mathrm{BE}$ or $\mathrm{E}$. $\mathrm{E}$ and $\mathrm{C}$ subjects were recruited from among the patients undergoing upper endoscopy in the same centres as BE patients and at the time the BE patients were identified.

For this study, BE was defined as a $15-\mathrm{mm}$ upward displacement of the squamocolumnar junction (Z-line) from the gastro-oesophageal junction at endoscopy, with histological confirmation of specialised intestinal metaplasia with 'goblet' cells $^{(12)}$. Interobserver variability in evaluating the length of columnar-lined oesophagus has been reported when BE segments $<1 \mathrm{~cm}$ are considered. In addition, intestinal metaplasia of the gastric cardia may be misclassified as short-segment BE. Therefore, we used a $15-\mathrm{mm}$ cut-off value to increase the accuracy of the BE diagnosis. At endoscopy, the Prague C \& M criteria were considered to define $\mathrm{BE}$ length ${ }^{(13)}$. Multiple biopsies of BE were taken, according to the Seattle Protocol ${ }^{(14)}$.

The E group was identified among patients with an endoscopic diagnosis of reflux $\mathrm{E}$ characterised by mucosal breaks. Grades A and B of the Los Angeles classification were considered $^{(15)}$. Patients in the E group underwent four biopsies: two at the Z-line and two at $2 \mathrm{~cm}$ above it.

Cases were consecutively recruited from eligible patients with a new diagnosis of BE or E from March 2009 to October 2012. The control group was consecutively recruited in the same units as the cases, through a non-random selection among eligible patients with no BE or E, undergoing upper endoscopy for any reason in the same period as the cases, both in presence or absence of GERD. Diagnosis of GERD was based on the presence of typical symptoms: heartburn or pyrosis (defined as retrosternal burning sensation, starting from the epigastric region and radiating up to the neck), regurgitation (as an acid or bitter taste in the mouth) and dysphagia. We defined as GERD positive those subjects reporting at least weekly heartburn and/or acid regurgitation
1 year before diagnosis ${ }^{(16)}$. Biopsies were interpreted in every centre by experienced gastro-intestinal pathologists.

In all cases, eligible patients were men or women, aged 18 years or older, able to give informed consent and agreeing to participate in a questionnaire, all without history of previous cancer or serious chronic diseases. The study was approved by the Ethical Committee of each centre and informed consent was obtained from all participating patients.

\section{Questionnaire}

The interviewers were centrally trained. The same questionnaire and coding manual were used for all subjects. Questions referred to symptoms or habits before the diagnosis of $\mathrm{BE}$ or $\mathrm{E}$ or before endoscopy for controls.

The questionnaire included questions on individual characteristics (education, occupation, weight and height), lifestyle habits (diet, tea, coffee and other types of beverage consumption, and smoking habit), past medical history, use of drugs, presence and duration of GERD symptoms (as the sum of the duration of at least weekly heartburn or regurgitation symptoms) and family history of cancer.

Each subject was asked to report about lifetime consumption of all alcoholic beverages: red and white wine, beer and liquors. To measure consumption of liquors three items were used: aperitifs and digestifs, containing up to $35 \%$ alcohol by volume, and spirits with more than $35 \%$.

For all beverages, subjects' entire drinking history was recalled in detail according to his/her drinking status, namely nondrinker, former drinker (who had quit at least 1 year before enrolment) and current drinker. Subjects were considered ever drinkers if they had consumed beverages at least monthly for 6 months or more. Questions were asked about the frequency of consumption, years of duration, age at initiation and, for former drinkers, years since cessation. One unit was equivalent to one glass of red or white wine (about $125 \mathrm{ml}$ ), one glass of lager or stout beer (one can or bottle, $330 \mathrm{ml}$ ) and one shot/glass of liquor ( $80 \mathrm{ml}$ for aperitifs and $40 \mathrm{ml}$ for digestifs or spirits).

\section{Statistical methods}

To estimate the effect of alcohol habit on the three-level health outcome (i.e. C group, E patients and BE patients) a multinomial logistic regression (MLR) modelling was applied ${ }^{(17)}$. MLR can be considered as an extension of the more widely used logistic regression modelling for binary outcome (i.e. ill cases $v$. healthy controls) in that it allows to assess the statistical association between health status and study exposure (i.e. alcoholrelated characteristics) performing simultaneously two binary comparisons: $\mathrm{E}$ patients $v$. $\mathrm{C}$ subjects and $\mathrm{BE}$ patients $v$. C subjects. Within each comparison, OR point estimate, along with corresponding $95 \% \mathrm{CI}$, is computed and considered as an index of association between each binary outcome (E $v$. C or BE $v . \mathrm{C}$ ) and each potential risk factor.

Alcohol habit is represented by several quantitative characteristics (frequency of consumption, number of units consumed, years of duration, age at initiation and years since cessation) each of which should be carefully considered and 
properly analysed in order to evaluate their distinct effect on individual health outcome. Such characteristics are generally well correlated and this may seriously impede a joint assessment through a regression modelling and, accordingly, prevent from controlling for the reciprocal confounding effect ${ }^{(18)}$. For these reasons, the following regression strategy was applied. Data were stratified according to drinking status (former and current drinkers) and in each stratum a MLR analysis was performed using non-drinkers as a reference category. Only one quantitative drinking variable (main predictor) at a time entered the regression equation after categorisation based on specific thresholds (percentiles) a priori defined on the distribution of the $\mathrm{C}$ group. The remaining quantitative characteristics, appropriately transformed (centred), entered the equation as continuous variables (covariates) ${ }^{(18)}$. In addition to alcoholrelated variables, all MLR included age at interview, sex, BMI, smoking habit, years of schooling, duration of GERD and categorical terms for collaborative centres.

The statistical significance (two-tailed $P<0.05$ ) was assessed using the likelihood-based $\chi^{2}$ test for linear trend (TLT) ${ }^{(17)}$. All statistical analyses were performed using STATA software (Release 13.1, 2013; StataCorp LP).

\section{Results}

\section{Baseline characteristics}

Characteristics of BE patients ( $n$ 339), E patients ( $n$ 462) and $\mathrm{C}$ subjects ( $n$ 619) are given in Table 1 . A total of 190 BE cases had also E with mean age $56 \cdot 2$ (sD 15.2) years for BE, $52 \cdot 6$ (SD 14.7) years for $\mathrm{E}$ and 53.7 (SD 14.1) years for C. Controls had a higher percentage of females and a lower BMI. C also had a lower percentage of smokers and a higher education qualification compared with the other groups. Reasons for endoscopy among C were mostly epigastric pain (38\%), regurgitation (25\%), dyspepsia (24\%), pyrosis or dysphagia (9\%), gastric or duodenal ulcer (3\%) and anaemia (1\%). According to our definition, GERD symptoms were present in $78.5 \%$ of $\mathrm{BE}$, $80.3 \%$ of $\mathrm{E}$ and in $54.8 \%$ of C. Among GERD-positive subjects, $80.8 \%$ of BE patients had suffered from symptoms for more than 3 years $v \cdot 50 \cdot 4 \%$ of $\mathrm{E}$ and $40.7 \%$ of $\mathrm{C}$.

\section{Red and white wine consumption}

Red wine was consumed by $72 \%$ of $\mathrm{BE}, 69.7 \%$ of $\mathrm{E}$ and $68.7 \%$ of $\mathrm{C}$. With respect to $\mathrm{C}, \mathrm{BE}$ patients were significantly more likely to be current drinkers ( $54 v .46 .8 \%, P=0.041$ ), drank more ( $\geq 6$ glasses/week: $35.4 v .27 \cdot 3 \%, P=0.012$ ) and for more time ( $>35$ years: 33.1 v. $24.9 \%, P=0.0092$ ). In addition, BE started drinking at earlier age ( $<25$ years: $26 \cdot 5 v .17 \cdot 4 \%, P=0 \cdot 010)$ and quitted drinking red wine later than $\mathrm{C}$ (time since cessation $\leq 3$ years: $4.4 v \cdot 1.5 \% \mathrm{C}, P=0 \cdot 011)$. A slightly higher percentage of current drinkers was present also among $\mathrm{E}$ patients (52.8\%, $P=0.053)$ who also drank more ( $\geq 6$ glasses/week: $37.2 \%, P=0.001)$ and started drinking at earlier age $(<25$ years: $25.3 \%, P=0.002)$ than $\mathrm{C}$. Instead, no differences were observed as for duration ( $>35$ years: $26 \%$ ) and time since cessation ( $\leq 3$ years: $2 \cdot 2 \%$ ).
Tables 2 and 3 show the results of MLR modelling reporting the risk of $\mathrm{BE}$ and $\mathrm{E}$ in former and current red wine drinkers, respectively, using non-wine drinkers as reference category. In current drinkers, main quantitative predictors were $a$ priori categorised according to quartile values of alcohol consumption among $\mathrm{C}$ subjects. In former drinkers, given the small number of subjects ( $n$ 66), categorisation was based on median values.

Overall, no clear, monotonic (increasing or decreasing) and statistically significant dose-response relationship was pointed out in both former and current drinkers. However, generalised U-shaped (non-linear) trend of $\mathrm{E} / \mathrm{BE}$ relative risk due to red wine consumption is noteworthy, particularly among current drinkers (Table 3; E $v$. C: models 2 and 3; BE $v$. C: all models), although such a tendency can be observed to some extent also in former drinkers (Table 2).

White wine was consumed by $54.3 \%$ of $\mathrm{BE}, 56.9 \%$ of $\mathrm{E}$ and $52.3 \%$ of C. E patients were significantly more likely to be current drinkers than $\mathrm{C}(41.8 v .34 .9 \%, P=0.025)$, whereas there was no difference between $\mathrm{C}$ and $\mathrm{BE}(39 \cdot 8 \%)$. The study subjects were similar with regard to frequency ( $\geq 6$ glasses/ week: BE $23.9 \%$, E $18.4 \%$ and C $20 \cdot 2 \%$ ). Subjects who had drunk for more time ( $>35$ years) were $18.4 \%$ in $\mathrm{C}, 23.9 \%$ in $\mathrm{BE}$ $(P=0.055)$ and $21 \%$ in $\mathrm{E}(P=0 \cdot 180)$. Both $\mathrm{BE}$ and $\mathrm{E}$ started drinking at younger age $(\leq 24$ years $=35.1$ and $34.9 v .28 \cdot 1 \%$, $P=0.030$ and 0.021 , respectively). Only a few subjects ( $n$ 44) were former drinkers $(5 \cdot 1 \%$ in $\mathrm{BE}, 2 \cdot 1 \%$ in $\mathrm{E}$ and $2.8 \%$ in $\mathrm{C})$ and this circumstance prevented us from performing further analyses on this subgroup. Table 4 shows the results of MLR analysis. Alcohol consumption variables were categorised according to quartiles (glasses/week and years of duration) and tertiles (age at initiation). No evidence of upward or downward trend in $\mathrm{E} / \mathrm{BE}$ risk was highlighted, but, as already seen for red wine consumption, a noteworthy U-shaped dose-response relationship was observed in at least four out of six cases (Table 4; E $v$. C: models 1 and 3; BE $v$. C: all models).

\section{Liquors and spirits consumption}

Overall, liquors/spirits were consumed by $42.5 \%$ of BE, $46 \cdot 8 \%$ of $\mathrm{E}$ and $38.6 \%$ of $\mathrm{C}$, with a significant difference between $\mathrm{E}$ and $\mathrm{C}(P=0.012)$. Percentage of current drinkers was lower among C $(8.9 \%)$ with respect to E $(17.3 \%, P<0.001)$ and $\mathrm{BE}(15.6 \%$, $P=0.0027)$. Both $\mathrm{BE}$ and $\mathrm{E}$ consumed these beverages for a longer time than $\mathrm{C}(\geq 15$ years: 17.7 and $12 \cdot 1 v .7 \cdot 6 \%, P<0.001$ and $P=0.017$, respectively). Furthermore, $\mathrm{BE}$ and $\mathrm{E}$ started consuming spirits at an earlier age ( $<20$ years: $8.6 \% \mathrm{BE}$, $6.1 \%$ E $v .3 .1 \%$ E, $P<0.001$ and $P=0.026$, respectively). With regard to the type of beverages, only a few subjects declared to drink aperitifs (18\% BE, 20.1\% E and 16.8\% C). Percentage of subjects consuming digestifs was similar between BE (28.6\%) and $\mathrm{C}(24.7 \%)$, whereas there was a higher percentage of $\mathrm{E}$ with respect to $\mathrm{C}(31.6 \%, P=0.015)$. Spirits consumption was reported by $30 \cdot 1 \%$ of $\mathrm{BE}, 26 \cdot 8 \%$ of $\mathrm{E}$ and $22 \cdot 1 \%$ of $\mathrm{C}$, with a significant difference between $\mathrm{BE}$ and $\mathrm{C}(P=0.009)$. Among these subjects a consumption frequency $\geq 1$ glass/week was reported by $15.9 \% \mathrm{BE}, 16.3 \% \mathrm{E}$ and $11.5 \% \mathrm{C}$, with a significant difference between $\mathrm{E}$ and $\mathrm{C}(P=0.030)$. Table 5 shows the results of MLR analysis performed on current drinkers solely 
Table 1. Characteristics of Barrett's oesophagus (BE), oesophagitis (E) and controls ( $n$ 1420) (Numbers and percentages)

\begin{tabular}{|c|c|c|c|c|c|c|c|}
\hline \multirow[b]{3}{*}{ Factors and levels } & \multicolumn{2}{|c|}{ Control } & \multicolumn{2}{|c|}{$E$} & \multicolumn{2}{|c|}{$\mathrm{BE}$} & \multirow[b]{3}{*}{$P$} \\
\hline & $n$ & $\%$ & $n$ & $\%$ & $n$ & $\%$ & \\
\hline & 619 & $100 \cdot 0$ & 462 & $100 \cdot 0$ & 339 & $100 \cdot 0$ & \\
\hline Sex & & & & & & & $<0.001$ \\
\hline Male & 252 & 40.7 & 285 & $61 \cdot 7$ & 229 & $67 \cdot 6$ & \\
\hline Female & 367 & 59.3 & 177 & 38.3 & 110 & $32 \cdot 4$ & \\
\hline Age at interview (years) & & & & & & & $<0.001$ \\
\hline$<41$ & 126 & $20 \cdot 4$ & 113 & 24.5 & 58 & $17 \cdot 1$ & \\
\hline $41-50$ & 129 & 20.8 & 92 & 19.9 & 66 & 19.5 & \\
\hline $51-60$ & 143 & $23 \cdot 1$ & 90 & 19.5 & 66 & 19.5 & \\
\hline $61-68$ & 129 & $20 \cdot 8$ & 101 & 21.9 & 58 & $17 \cdot 1$ & \\
\hline$>68$ & 93 & $15 \cdot 0$ & 66 & $14 \cdot 3$ & 91 & $26 \cdot 8$ & \\
\hline Smoking status & & & & & & & 0.001 \\
\hline Never smoker & 330 & 53.3 & 218 & $47 \cdot 2$ & 135 & 39.8 & \\
\hline Former smoker & 156 & $25 \cdot 2$ & 138 & 29.9 & 126 & 37.2 & \\
\hline Years since cessation $\leq 15$ & 86 & 13.9 & 83 & 18.0 & 55 & $16 \cdot 2$ & \\
\hline Years since cessation $>15$ & 70 & 11.3 & 55 & 11.9 & 71 & 20.9 & \\
\hline Cigarettes $/ \mathrm{d} \leq 13$ & 71 & 11.5 & 51 & 11.0 & 57 & $16 \cdot 8$ & \\
\hline Cigarettes $/ \mathrm{d}>13$ & 85 & 13.7 & 87 & $18 \cdot 8$ & 69 & 20.4 & \\
\hline Current smoker & 133 & 21.5 & 106 & 22.9 & 78 & $23 \cdot 0$ & \\
\hline Cigarettes $/ \mathrm{d} \leq 13$ & 72 & 11.6 & 45 & $9 \cdot 7$ & 38 & 11.2 & \\
\hline Cigarettes $/ \mathrm{d}>13$ & 61 & 9.9 & 61 & $13 \cdot 2$ & 40 & 11.8 & \\
\hline Wine drinking habit & & & & & & & 0.001 \\
\hline Never drinker & 169 & $27 \cdot 3$ & 114 & 24.7 & 77 & $22 \cdot 7$ & \\
\hline Former drinker & 29 & 4.7 & 16 & 3.5 & 29 & 8.6 & \\
\hline Current drinker & 304 & 49.1 & 260 & $56 \cdot 3$ & 194 & $57 \cdot 2$ & \\
\hline Ever drinker & 117 & $18 \cdot 9$ & 72 & $15 \cdot 6$ & 39 & 11.5 & \\
\hline $\mathrm{BMI}\left(\mathrm{kg} / \mathrm{m}^{2}\right)$ & & & & & & & $<0.001$ \\
\hline$\leq 22.27$ & 157 & $25 \cdot 4$ & 64 & 13.9 & 60 & $17 \cdot 7$ & \\
\hline $22 \cdot 28-24 \cdot 22$ & 148 & $23 \cdot 9$ & 86 & $18 \cdot 6$ & 65 & $19 \cdot 2$ & \\
\hline $24.23-25.95$ & 115 & 18.6 & 98 & 21.2 & 60 & $17 \cdot 7$ & \\
\hline $25.96-28.09$ & 104 & $16 \cdot 8$ & 103 & $22 \cdot 3$ & 76 & $22 \cdot 4$ & \\
\hline$>28.09$ & 95 & $15 \cdot 3$ & 111 & 24.0 & 78 & 23.0 & \\
\hline Duration of GERD & & & & & & & $<0.001$ \\
\hline Never & 280 & $45 \cdot 2$ & 91 & $19 \cdot 7$ & 73 & 21.5 & \\
\hline$<3$ years & 201 & 32.5 & 184 & 39.8 & 51 & $15 \cdot 0$ & \\
\hline $3-10$ years & 92 & 14.9 & 142 & 30.7 & 127 & 37.5 & \\
\hline$>10$ years & 46 & $7 \cdot 4$ & 45 & $9 \cdot 7$ & 88 & $26 \cdot 0$ & \\
\hline Years of schooling & & & & & & & $<0.001$ \\
\hline$<6$ & 193 & 31.2 & 104 & 22.5 & 96 & $28 \cdot 3$ & \\
\hline $6-8$ & 216 & 34.9 & 145 & 31.4 & 85 & $25 \cdot 1$ & \\
\hline $9-13$ & 183 & 29.6 & 171 & 37.0 & 116 & $34 \cdot 2$ & \\
\hline$>13$ & 27 & 4.4 & 42 & $9 \cdot 1$ & 42 & $12 \cdot 4$ & \\
\hline
\end{tabular}

GERD, gastro-oesophageal reflux disease.

because of the limited information available on former drinkers. In this context, MLR failed to point out any apparent linear trend in risk by alcohol consumption. However, higher alcohol consumption levels ( $>1$ glass/week, $>15$ years of duration and $<30$ years of age at initiation) seemed to bring about some risk excesses, for both adverse health outcomes, ranging from $1 \cdot 14$ to $2 \cdot 30$, although statistically non-significant.

\section{Beer consumption}

Beer was currently consumed by $52.8 \%$ of BE, $58.7 \%$ of $\mathrm{E}$ and $50.7 \%$ of $\mathrm{C}$, with a significant difference between $\mathrm{C}$ and $\mathrm{E}$ $(P=0.011)$. A higher consumption ( $\geq 6$ glasses/week) was reported by $22.7 \%$ of $\mathrm{BE}, 24.5 \%$ of $\mathrm{E}$ and $21.2 \%$ of $\mathrm{C}$. E patients differed significantly from $\mathrm{C}$ also with regard to duration of intake ( $>20$ years: $18.4 v .11 .8 \%, P<0 \cdot 010$ ), whereas there was no difference between $\mathrm{C}$ and $\mathrm{BE}(15 \cdot 7 \%)$. Moreover, in this case, given the lack of information about former drinkers, regression analysis was restricted to current drinkers (Table 6). Noteworthy and statistically significant decreasing doseresponse relationships were found in BE patients for frequency (model 1, TLT $P=0.002$ ) and duration of beer consumption (model 2; TLT $P=0.009$ ). Similar but less clear downward tendencies were also found for $\mathrm{E}$ patients (model 1, TLT $P=0 \cdot 801 ;$ model 2, TLT $P=0 \cdot 078$ ).

\section{Discussion}

In this study we evaluated the association between consumption of alcoholic beverages (wine, beer, liquors) and the presence of $\mathrm{BE}$ or reflux $\mathrm{E}$, compared with a control group of non-neoplastic patients undergoing endoscopy for any reason, but with no BE or E. 
Table 2. Relative risk of Barrett's oesophagus (BE) and oesophagitis $(E)$ according to red wine drinking habit estimated through multinomial logistic regression modelling among former drinkers using never drinkers as a reference*

(Odds ratios and $95 \%$ confidence intervals)

\begin{tabular}{|c|c|c|c|c|c|c|}
\hline \multirow[b]{3}{*}{ Model } & \multirow{2}{*}{\multicolumn{2}{|c|}{ Red wine drinking characteristics }} & \multicolumn{4}{|c|}{ Former drinkers $(n 66) v$. non-red wine drinkers ( $n$ 429) } \\
\hline & & & \multicolumn{2}{|c|}{ E v. C } & \multicolumn{2}{|c|}{$\mathrm{BE} v . \mathrm{C}$} \\
\hline & Covariates & Main predictor & OR & $95 \% \mathrm{Cl}$ & OR & $95 \% \mathrm{Cl}$ \\
\hline \multirow[t]{5}{*}{1} & Years of duration & Glasses/d & & & & \\
\hline & Age at initiation & Non-drinker & 1.00 & Ref. & 1.00 & Ref. \\
\hline & Years since cessation & $\leq 1$ & 0.48 & $0.18,1.31$ & 0.86 & $0.36,2.02$ \\
\hline & & $>1$ & 0.56 & $0.08,4.11$ & 1.89 & $0.30,11.84$ \\
\hline & & TLT & \multicolumn{2}{|c|}{0.159} & \multicolumn{2}{|c|}{0.159} \\
\hline \multirow[t]{5}{*}{2} & Glasses/d & Years of duration & & & & \\
\hline & Age at initiation & Non-drinker & 1.00 & Ref. & 1.00 & Ref. \\
\hline & Years since cessation & $\leq 31$ & 0.41 & $0.02,10.58$ & 1.12 & $0.06,21.06$ \\
\hline & & $>31$ & 0.33 & $0.01,10.94$ & 1.57 & $0.07,35.39$ \\
\hline & & TLT & \multicolumn{2}{|c|}{0.608} & 0.663 & \\
\hline \multirow[t]{5}{*}{3} & Glasses/d & Age at initiation & & & & \\
\hline & Years since cessation & Non-drinker & 1.00 & Ref. & 1.00 & Ref. \\
\hline & Years of duration & $>25$ & 0.50 & $0.17,1.44$ & 1.09 & $0.42,2 \cdot 80$ \\
\hline & & $\leq 25$ & 0.74 & $0.22,2.51$ & 0.71 & $0.21,2 \cdot 41$ \\
\hline & & TLT & \multicolumn{2}{|l|}{0.326} & \multicolumn{2}{|c|}{0.661} \\
\hline \multirow[t]{5}{*}{4} & Glasses/d & Years since cessation & & & & \\
\hline & Age at initiation & Non-drinker & 1.00 & Ref. & 1.00 & Ref. \\
\hline & Years of duration & $>3$ & 0.20 & $0.05,0.77$ & 0.39 & $0 \cdot 13,1 \cdot 18$ \\
\hline & & $\leq 3$ & 0.96 & $0 \cdot 29,3 \cdot 15$ & $2 \cdot 01$ & $0.72,5 \cdot 66$ \\
\hline & & TLT & \multicolumn{2}{|c|}{0.263} & \multicolumn{2}{|c|}{0.614} \\
\hline
\end{tabular}

C, control; Ref., referent values; GERD, gastro-oesophageal reflux disease; TLT, test for linear trend. $P$ of the likelihood-based $\chi^{2}$ test for linear trend

* OR (relative risk) point estimate, adjusted for age at interview, sex, BMI, smoking habit, years of schooling, duration of GERD and collaborative centre.

Table 3. Relative risk of Barrett's oesophagus (BE) and oesophagitis (E) according to red wine drinking habit estimated through multinomial logistic regression modelling among current drinkers using never drinkers as a reference*

(Odds ratios and $95 \%$ confidence intervals)

\begin{tabular}{|c|c|c|c|c|c|c|}
\hline \multirow[b]{3}{*}{ Model } & \multirow{2}{*}{\multicolumn{2}{|c|}{ Red wine drinking characteristics }} & \multicolumn{4}{|c|}{ Current drinkers ( $n$ 717) v. non-red wine drinkers ( $n$ 429) } \\
\hline & & & \multicolumn{2}{|c|}{ E v. C } & \multicolumn{2}{|c|}{$\mathrm{BE} v . \mathrm{C}$} \\
\hline & Covariates & Main predictor & OR & $95 \% \mathrm{Cl}$ & OR & $95 \% \mathrm{Cl}$ \\
\hline \multirow[t]{7}{*}{1} & Years of duration & Glasses/week & & & & \\
\hline & Age at initiation & Non-drinker & 1.00 & Ref. & 1.00 & Ref. \\
\hline & & $<4$ & 0.70 & $0.43,1.16$ & 0.65 & $0.36,1 \cdot 15$ \\
\hline & & $4-5$ & 0.70 & $0.44,1.13$ & 0.83 & $0.50,1.38$ \\
\hline & & 6 & 1.32 & $0.87,2.01$ & 1.11 & $0.69,1.80$ \\
\hline & & $>6$ & 0.64 & $0.40,1.03$ & $0 \cdot 71$ & $0.41,1.21$ \\
\hline & & TLT & & & & \\
\hline \multirow[t]{7}{*}{2} & Glasses/week & Years of duration & & & & \\
\hline & Age at initiation & Non-drinker & 1.00 & Ref. & 1.00 & Ref. \\
\hline & & $<24$ & 1.09 & $0.64,1.83$ & 1.08 & $0.58,2.00$ \\
\hline & & $24-35$ & 0.85 & $0.53,1.38$ & 0.85 & $0.50,1.46$ \\
\hline & & $36-46$ & 0.72 & $0.45,1.16$ & 0.55 & $0.31,0.95$ \\
\hline & & $>46$ & 0.85 & $0.48,1.52$ & 0.94 & $0.51,1.76$ \\
\hline & & TLT & & & & \\
\hline \multirow[t]{7}{*}{3} & Glasses/week & Age at initiation & & & & \\
\hline & Years of duration & Non-drinker & 1.00 & Ref. & 1.00 & Ref. \\
\hline & & $>25$ & 0.87 & $0.49,1.57$ & 0.68 & $0.35,1.31$ \\
\hline & & $25-19$ & 0.76 & $0.52,1.12$ & 0.69 & $0.44,1.08$ \\
\hline & & $18-16$ & 1.28 & $0.76,2.13$ & 1.31 & $0.74,2.32$ \\
\hline & & $<16$ & 0.86 & $0.52,1.42$ & 1.04 & $0.59,1.82$ \\
\hline & & TLT & & & & \\
\hline
\end{tabular}

C, control; Ref., referent values; GERD, gastro-oesophageal reflux disease; TLT, test for linear trend. $P$ of the likelihood-based $\chi^{2}$ test for linear trend.

* OR (relative risk) point estimate, adjusted for age at interview, sex, BMl, smoking habit, years of schooling, duration of GERD and collaborative centre. 
Table 4. Relative risk of Barrett's oesophagus (BE) and oesophagitis $(E)$ according to white wine drinking habit estimated through multinomial logistic regression modelling among current drinkers using never drinkers as a reference* (Odds ratios and $95 \%$ confidence intervals)

\begin{tabular}{|c|c|c|c|c|c|c|}
\hline \multirow[b]{3}{*}{ Model } & \multirow{2}{*}{\multicolumn{2}{|c|}{ White wine drinking characteristics }} & \multicolumn{4}{|c|}{ Current drinkers $(n 544) v$. non-white wine drinkers ( $n$ 649) } \\
\hline & & & \multicolumn{2}{|c|}{$\mathrm{E} v \cdot \mathrm{C}$} & \multicolumn{2}{|c|}{$\mathrm{BE} v . \mathrm{C}$} \\
\hline & Covariates & Main predictor & OR & $95 \% \mathrm{Cl}$ & OR & $95 \% \mathrm{Cl}$ \\
\hline \multirow[t]{7}{*}{1} & Years of duration & Glasses/week & & & & \\
\hline & Age at initiation & Non-drinker & 1.00 & Ref. & 1.00 & Ref. \\
\hline & & $<4$ & 0.77 & $0.49,1.22$ & 0.68 & $0.40,1.16$ \\
\hline & & $4-5$ & 0.76 & $0.42,1.39$ & 1.34 & $0.73,2.48$ \\
\hline & & 6 & 0.85 & $0.51,1.44$ & 1.01 & $0.58,1.79$ \\
\hline & & $>6$ & 1.01 & $0.60,1.69$ & 0.81 & $0.45,1.46$ \\
\hline & & TLT & \multicolumn{2}{|c|}{0.686} & \multicolumn{2}{|c|}{0.984} \\
\hline \multirow[t]{7}{*}{2} & Glasses/week & Years of duration & & & & \\
\hline & Age at initiation & Non-drinker & 1.00 & Ref. & 1.00 & Ref. \\
\hline & & $<24$ & 0.92 & $0.53,1.60$ & 0.74 & $0.37,1.46$ \\
\hline & & $24-35$ & 0.73 & $0.41,1.29$ & 0.91 & $0.50,1.66$ \\
\hline & & $36-46$ & 1.05 & $0.63,1.74$ & 0.71 & $0.38,1.32$ \\
\hline & & $>46$ & 0.67 & $0.37,1.22$ & 1.20 & $0.66,2 \cdot 18$ \\
\hline & & TLT & \multicolumn{2}{|c|}{0.259} & \multicolumn{2}{|c|}{0.876} \\
\hline \multirow[t]{6}{*}{3} & Glasses/week & Age at initiation & & & & \\
\hline & Years of duration & Non-drinker & 1.00 & Ref. & 1.00 & Ref. \\
\hline & & $>24$ years & 0.85 & $0.48,1.50$ & 0.63 & $0.33,1.22$ \\
\hline & & $24-18$ years & 0.83 & $0.55,1.25$ & 0.79 & $0.49,1.27$ \\
\hline & & $<18$ years & 0.85 & $0.53,1.36$ & $1 \cdot 27$ & $0.77,2.09$ \\
\hline & & TLT & \multicolumn{2}{|c|}{0.319} & \multicolumn{2}{|c|}{0.817} \\
\hline
\end{tabular}

C, control; Ref., referent values; GERD, gastro-oesophageal reflux disease; TLT, test for linear trend. $P$ of the likelihood-based $\chi^{2}$ test for linear trend.

${ }^{*}$ OR (relative risk) point estimate, adjusted for age at interview, sex, BMI, smoking habit, years of schooling, duration of GERD and collaborative centre.

Table 5. Relative risk of Barrett's oesophagus and oesophagitis according to heavy alcohol consumption habit estimated through multinomial logistic regression modelling among current drinkers using never drinkers as a reference* (Odds ratios and $95 \%$ confidence intervals)

\begin{tabular}{|c|c|c|c|c|c|c|}
\hline \multirow[b]{3}{*}{ Model } & \multirow{2}{*}{\multicolumn{2}{|c|}{ Liquors/spirits drinking characteristics }} & \multicolumn{4}{|c|}{ Current drinkers $(n 188) v$. non-spirits drinkers ( $n$ 821) } \\
\hline & & & \multicolumn{2}{|c|}{ E v. C } & \multicolumn{2}{|c|}{$\mathrm{BE} v . \mathrm{C}$} \\
\hline & Covariates & Main predictor & OR & $95 \% \mathrm{Cl}$ & OR & $95 \% \mathrm{Cl}$ \\
\hline \multirow[t]{6}{*}{1} & Years of duration & Glasses/week & & & & \\
\hline & Age at initiation & Non-drinker & 1.00 & Ref. & 1.00 & Ref. \\
\hline & & $<1$ & 0.65 & $0.21,2.00$ & 2.23 & $0.86,5.79$ \\
\hline & & 1 & 0.89 & $0.45,1 \cdot 76$ & 0.67 & $0.31,1.45$ \\
\hline & & $>1$ & $2 \cdot 30$ & $0.94,5.62$ & $1 \cdot 28$ & $0.44,3.77$ \\
\hline & & TLT & & & & \\
\hline \multirow[t]{6}{*}{2} & Glasses/week & Years of duration & & & & \\
\hline & Age at initiation & Non-drinker & 1.00 & Ref. & 1.00 & Ref. \\
\hline & & $<15$ & 1.83 & $0.87,3.84$ & 0.94 & $0.39,2 \cdot 28$ \\
\hline & & $15-29$ & $1 \cdot 14$ & $0.53,2.47$ & 1.33 & $0.60,2.98$ \\
\hline & & $>29$ & 0.99 & $0.48,2.03$ & 1.25 & $0.59,2.68$ \\
\hline & & TLT & & & & \\
\hline \multirow[t]{6}{*}{3} & Glasses/week & Age at initiation & & & & \\
\hline & Years of duration & Non-drinker & 1.00 & Ref. & 1.00 & Ref. \\
\hline & & $>30$ & 0.99 & $0.49,2.00$ & 0.96 & $0.43,2.18$ \\
\hline & & $30-20$ & 1.73 & $0.88,3.40$ & $1 \cdot 18$ & $0.54,2.58$ \\
\hline & & $<20$ & $1 \cdot 17$ & $0.50,2.72$ & 1.33 & $0.57,3.11$ \\
\hline & & TLT & & & & \\
\hline
\end{tabular}

C, control; Ref., referent values; GERD, gastro-oesophageal reflux disease; TLT, test for linear trend. $P$ of the likelihood-based $\chi^{2}$ test for linear trend.

* OR (relative risk) point estimate, adjusted for age at interview, sex, BMI, smoking habit, years of schooling, duration of GERD and collaborative centre.

We observed some risk excesses for both pathologies with a higher intake of any type of liquors/spirits, nevertheless, no apparent linear trends by alcohol consumption categories were found.
Interestingly, no evident monotonic dose-response relationship was found with wine consumption. In particular, using restricted cubic splines ${ }^{(19)}$ generalised U-shaped (non-linear) trends of $\mathrm{E} / \mathrm{BE}$ risk by consumption of both red and white wine 
Table 6. Relative risk of Barrett's oesophagus (BE) and oesophagitis $(E)$ according to beer drinking habit estimated through multinomial logistic regression modelling among current drinkers using never drinkers as a reference*

(Odds ratios and $95 \%$ confidence intervals)

\begin{tabular}{|c|c|c|c|c|c|c|}
\hline \multirow[b]{3}{*}{ Model } & \multirow{2}{*}{\multicolumn{2}{|c|}{ Beer drinking characteristics }} & \multicolumn{4}{|c|}{ Current drinkers ( $n$ 288) v. non-beer drinkers ( $n$ 656) } \\
\hline & & & \multicolumn{2}{|c|}{$\mathrm{E} v . \mathrm{C}$} & \multicolumn{2}{|c|}{$\mathrm{BE} v . \mathrm{C}$} \\
\hline & Covariates & Main predictor & OR & $95 \% \mathrm{Cl}$ & OR & $95 \% \mathrm{Cl}$ \\
\hline \multirow[t]{7}{*}{1} & Years of duration & Glasses/week & & & & \\
\hline & & Non-drinker & 1.00 & Ref. & 1.00 & Ref. \\
\hline & & $<4$ & 0.43 & $0.13,1.48$ & 0.66 & $0.20,2 \cdot 13$ \\
\hline & & $4-5$ & $1 \cdot 21$ & $0.53,2.77$ & $0 \cdot 80$ & $0.28,2.24$ \\
\hline & & 6 & 0.54 & $0.32,0.90$ & 0.37 & $0.20,0.68$ \\
\hline & & $>6$ & 0.79 & $0.39,1.58$ & 0.45 & $0.17,1.16$ \\
\hline & & TLT & \multicolumn{2}{|c|}{0.801} & \multicolumn{2}{|c|}{0.002} \\
\hline \multirow[t]{6}{*}{2} & Glasses/week & Years of duration & & & & \\
\hline & & Non-drinker & 1.00 & Ref. & 1.00 & Ref. \\
\hline & & $<21$ & 0.76 & $0.40,1.45$ & 0.41 & $0.18,0.95$ \\
\hline & & $21-30$ & 0.53 & $0.29,0.99$ & 0.44 & $0.21,0.90$ \\
\hline & & $>30$ & 0.73 & $0.40,1.34$ & 0.52 & $0.26,1.05$ \\
\hline & & TLT & \multicolumn{2}{|c|}{0.078} & \multicolumn{2}{|c|}{0.009} \\
\hline
\end{tabular}

C, control; Ref., referent values; GERD, gastro-oesophageal reflux disease; TLT, test for linear trend. $P$ of the likelihood-based $\chi^{2}$ test for linear trend.

${ }^{\star}$ OR (relative risk) point estimate, adjusted for age at interview, sex, BMI, smoking habit, years of schooling, duration of GERD and collaborative centre.

were pointed out: a beneficial effect seems to occur with moderate intake or shorter duration, whereas worse outcomes resulted for non-wine drinkers and heavy/longer drinking habit (Fig. 1).

In addition, beer consumers were found to be at lower risk of $\mathrm{BE}$ and, to a lesser degree, of $\mathrm{E}$, irrespective of frequency or duration. It is noteworthy that a similar response was shown in the majority of surveys examining the relationship between alcohol consumption and multiple cardiovascular outcomes ${ }^{(20-22)}$. According to a study, there was a U-shaped relationship between beer, wine and spirits intake, and heart failure incidence, with a nadir at low-to-moderate intake. In this case, wine and spirits no longer appeared protective above 7 drinks/week, whereas beer appeared potentially protective for 7-14 drinks/week ${ }^{(23)}$. On the other hand, occasional or chronic ethanol intake at high levels increases the risk for myocardial infarction and stroke ${ }^{(24)}$.

Alcohol is an established risk factor for oesophageal squamous cell carcinoma ${ }^{(6)}$, but reports about the association between alcohol and reflux E and BE, as well as EAC are still inconsistent. Alcohol consumption may increase gastro-oesophageal reflux symptoms, cause damage to the oesophageal mucosa and/or promote carcinogenesis ${ }^{(25)}$. Several studies have demonstrated an association between alcohol intake and increased GERD symptoms ${ }^{(26-29)}$, nevertheless, these results are not corroborated by some authors ${ }^{(30)}$ or an inverse association was observed ${ }^{(31)}$. A higher total alcohol intake was found among EAC patients compared with controls, although the risk of tumour was quite lower than the risk of squamous cell carcinoma ${ }^{(32-34)}$. On the contrary, other authors reported an inverse association with moderate intake ${ }^{(35-37)}$, whereas no effect was found in other studies $^{(38-44)}$. Little is known about the real effect of alcohol on $\mathrm{BE}$, particularly with regard to alcohol types. Again, a certain number of studies found no relationship ${ }^{(3,8,9,16,43,45-47)}$, but lifetime alcohol intake was associated with lower BE risk according to some reports ${ }^{(9,44)}$. Thrift et al. ${ }^{(48)}$ found inverse associations with intermediate or higher levels of lifetime total alcohol consumption, for comparisons with both population and inflammation controls. Conversely, alcohol was found to increase the risk only when comparing cases with GERD controls according to Kubo et al. ${ }^{(10)}$. In a recent pooled analysis of data from five case-control studies, a borderline significant inverse association between $\mathrm{BE}$ and any alcohol consumption was found when cases were compared with population controls, although the risk did not decrease in a linear manner. In this contest, subjects consuming 3 to $<5$ drinks/d had about half the risk of BE compared with non-drinkers, but there were no statistically significant associations with lower or higher levels of alcohol. No association with alcohol was found when cases were compared with GERD controls ${ }^{(7)}$.

Considering alcohol type, a modest intake of red wine has been associated with a reduced risk of EAC in some reports ${ }^{(35,49,50)}$, but not in a cohort study ${ }^{(42)}$. An inverse association was found also with $\mathrm{BE}^{(45,46)}$, even when there was no association with total alcohol use ${ }^{(10)}$.

The role of beer consumption is contradictory, too. The highest intake of beer at younger age was inversely related with $\mathrm{BE}$, but the association was no more present when considering consumption 5 years before the interview according to Anderson et al. ${ }^{(43)}$. Thrift et al. ${ }^{(48)}$ found an inverse linear trend with beer consumption using both population and inflammation controls, whereas BE patients were up 2-fold more likely to drink beer when compared with GERD controls in another survey ${ }^{(10)}$.

A few studies reported an adverse effect of liquor consumption. Thrift et al. ${ }^{(48)}$ observed a significant linear trend for increasing risk of dysplastic BE with rising liquor consumption by comparing $\mathrm{BE}$ cases with inflammation controls. A high liquor consumption ( $\geq 40$ drinks/month) was also associated with an increased risk of both GERD and BE when patients were compared with asymptomatic individuals. In this case, the risk of $\mathrm{BE}$ increased 3-fold ${ }^{(38)}$.

Most reports on the relationship between alcohol consumption and $\mathrm{E}$ are from Asian areas where consumption of different 

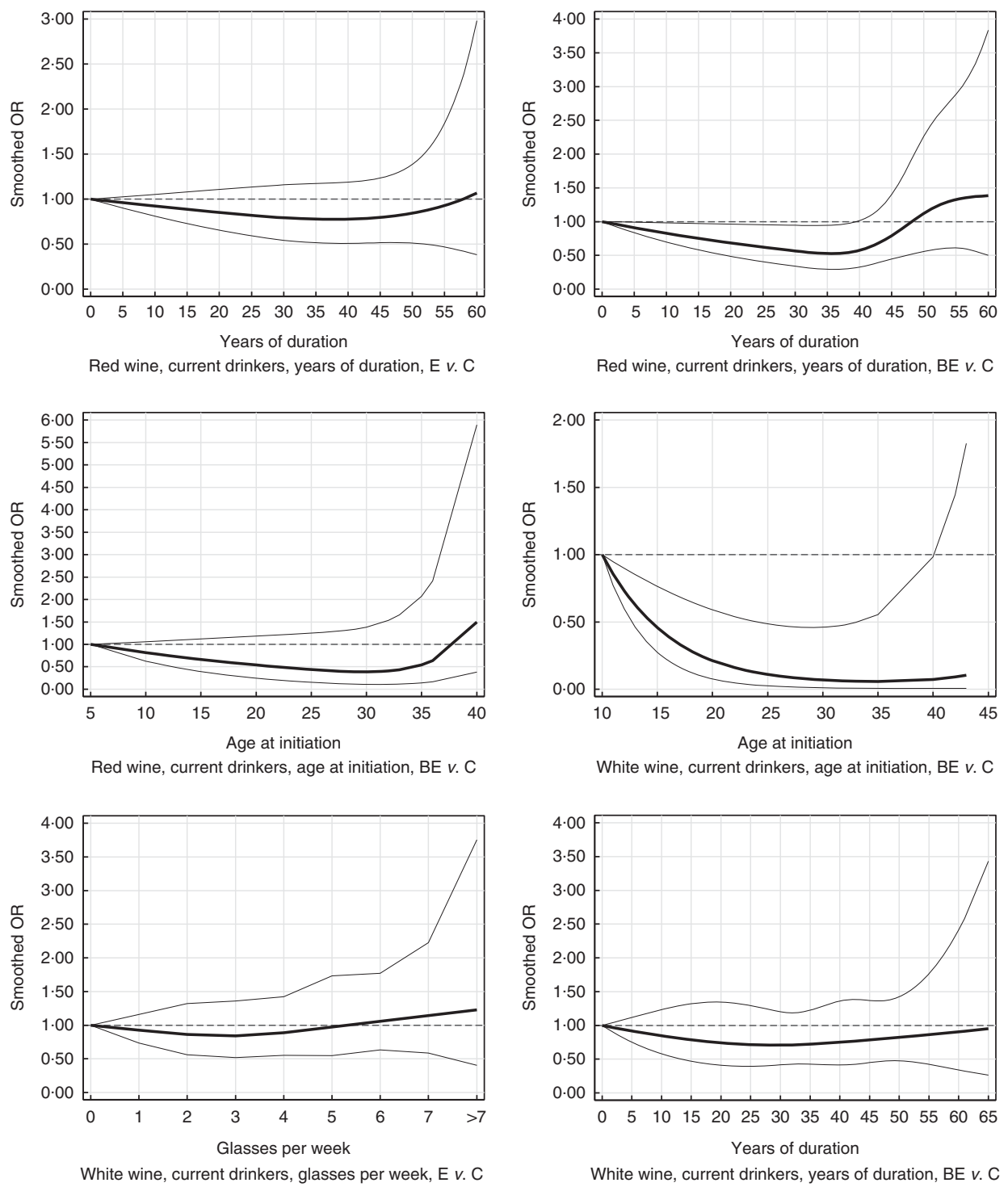

Fig. 1. U-shaped dose-response relationships between risk of oesophagitis (E)/Barrett's oesophagus (BE) and level of red and white wine consumption in current drinkers compared with non-wine drinkers. Smoothed OR based on three/four-knot restricted cubic splines. C, control.

beverages could not be representative of that in Western countries. Alcohol consumption was positively correlated with both reflux $\mathrm{E}$ and non-erosive reflux disease in a large Japanese cohort of subjects who underwent upper gastro-intestinal endoscopy, compared with GERD-free subjects ${ }^{(51)}$. Total alcohol consumption did not seem to be a risk factor of $\mathrm{E}$ according to a number of Western authors ${ }^{(3,52,53)}$, nevertheless, Anderson et $a l .{ }^{(43)}$ found that alcohol consumption at least once per month in early adulthood may increase more than 2-fold the risk of developing $\mathrm{E}$. In this case, nevertheless, there was an inverse association when the alcohol intake increased. In addition, a regular alcohol intake was found to increase about $70 \%$ of the risk of any grade of $\mathrm{E}$ in a prospective cohort study on patients with heartburn ${ }^{(54)}$.

When considering alcohol type, the risk of $\mathrm{E}$ appeared to increase up to 2-fold with a high liquor intake before diagnosis ${ }^{(43,45)}$, whereas subjects with higher wine intake had half the risk of the disease compared with nondrinkers ${ }^{(43)}$.

The data on the association between $\mathrm{E}$ and beer are very scarce. Anderson et al. ${ }^{(43)}$ showed that the above-reported results seen for the overall alcohol intake in early adulthood could be explained by beer consumption and that there was no association when beer was consumed 5 years before the interview date.

To date, no clear hypotheses exist on the association between alcoholic beverages and $\mathrm{BE}$ or E; nevertheless, some mechanisms may support the hypothesis of a negative or protective effect. Polyphenols, in particular resveratrol, present in red grape skin may reduce the oxidative damage caused by GERD, thereby decreasing the risk of $\mathrm{E}$ and $\mathrm{BE}$, as well as of $\mathrm{EAC}^{(55-58)}$. Antioxidants are also present to a lesser extent in beer ${ }^{(59)}$ and this could explain the benefits of beer consumption as observed in some reports ${ }^{(48)}$. It has also been suggested that the protective effects of ethanol consumption may also arise from reductions in 
insulin resistance or increased levels of lipoproteins ${ }^{(60)}$, but it could be that the apparent health benefits of moderate alcohol consumption and wine drinking may be due to other protective unmeasured or unknown lifestyle habits of moderate drinkers $v$. abstainers or to favourable risk profiles in moderate drinkers ${ }^{(61)}$. On the other hand, as emphasised by other authors, unlike wine drinkers, liquor drinkers are less likely to consume these beverages with food, thereby increasing the possibility of irritation and damage to the oesophageal tissues ${ }^{(10)}$.

The divergent results among studies might derive from heterogeneity of the cases or reference groups examined in many surveys (incident or prevalent cases, population controls without endoscopy, GERD controls) that might have influenced the individual behavioural patterns ${ }^{(16)}$. The lack of information on the presence of $\mathrm{GERD}^{(8)}$ and the use of population controls may not provide sufficient number of GERD patients to estimate the effect of a risk factor independent of GERD symptoms ${ }^{(10)}$. In addition, controls sampled from the general population might have an undiagnosed $\mathrm{BE}$ or GERD, even if $\mathrm{BE}$ is not frequent in endoscopy series of healthy volunteers and has been diagnosed in $<10 \%$ of patients with severe reflux undergoing endoscopy ${ }^{(62,63)}$.

Also, contrasting results may be obtained when adjustment is made for possible confounders or with different temporalities of the associations; that is, a lifetime or recent beverage consumption and timing of the intake in relationship to diagnosis (cases or GERD controls may have consumed more alcohol in early life, reducing their intake later because of symptoms or diagnosis of oesophageal abnormality $)^{(10,43,48,64)}$.

Method of ascertaining alcohol consumption and definition of drinking (i.e. frequency of servings, quantity, ethanol content) may also be a limitation in comparing studies ${ }^{(9,43)}$.

One of the strengths of present survey is the fact that, to the best of our knowledge, this is the first study analysing the association between $\mathrm{BE}$ or $\mathrm{E}$ and drinking habits in a pool of Italian areas. In this study, controls did not seem to differ from the Italian population in terms of their alcohol consumption and it is worth mentioning that it is possible that the type and amount of alcohol-containing beverages as consumed by Italians differ from those in other studies. Furthermore, both cases and controls were endoscopically documented, cases had no prior diagnosis of $\mathrm{BE}$ or $\mathrm{E}$ and we had the possibility of controlling for the presence and the length of GERD duration. On the other hand, information on symptoms was self-reported. The recording of exposure may vary depending on the investigator's knowledge of an individual's disease status, but in this case interviewers knew that patients (both cases and controls) might have had GERD symptoms, but were unaware of the subjects diagnosis.

A potential bias is the fact that subjects with symptoms or suspicion of BE or E may have avoided alcohol because it could exacerbate their symptoms. These subjects are more likely to be diagnosed with $\mathrm{BE}$ or $\mathrm{E}$ having a more health-seeking behaviour, nevertheless, in our series the number of former drinkers was quite low and also controls had endoscopy because of gastric or oesophageal problems. Generally, a recall bias might have influenced individuals' drinking history, with high consumers of alcohol reporting a lower intake.
Another limitation is the use of the analyses of non-drinkers instead of abstainers as the comparison group. Nevertheless, results did not substantially change when using abstainers as the reference category because of the quite low number in this group (for a total of ninety subjects). In addition, we did not report results for total alcohol intake for subjects drinking both red and white wine as a clear overestimate of alcohol consumption was obtained.

Selection of controls was non-random; nevertheless, we think there was no non-response bias or a different reporting of exposure between cases and controls, as it is generally recognised that some dietary habits (and alcohol consumption) may be risk factors for digestive diseases. On the other hand, controls were younger than cases: this aspect and the fact that they did not represent an asymptomatic population may have resulted in an underestimation of the strength of the association between exposure (alcohol) and outcome.

Although we had information on dietary habits of both cases and controls, we did not consider them in this analysis as diet is an extremely complex mix of several characteristics (foods, servings, macro- and micro-nutrients, dietary patterns, etc.), which can show different effects on human health. Hence, even if we had taken into consideration one or more dietary patterns as potential confounding factors it would have not ruled out residual confounding factors anyway.

Actually, different components of diet or dietary habits can modulate the association with alcohol intake and oesophageal diseases, both leading to an increased risk or acting as protective factors ${ }^{(65)}$. Moreover, as outlined by some authors, the frequency of general alcohol consumption and type of beverage are related to many factors; as wine drinkers may have different (and more probably healthier) lifestyles and dietary habits compared with beer and liquor drinkers ${ }^{(10,66)}$. Nevertheless, adjusting for fruit and vegetable intake and for most of the factors that were associated with alcohol preference did not attenuate the inverse association for wine drinkers compared with non-drinkers in a study from Kubo et al. ${ }^{(10)}$.

In conclusion, consistent with findings from other studies, our results did not support a causative role of alcohol in aetiogenesis of BE or E. Although our results were often not statistically significant, data suggested a reduced risk of $\mathrm{BE}$ and $\mathrm{E}$ with a low intake of wine and with beer consumption. A nonsignificant increased risk for both $\mathrm{BE}$ and $\mathrm{E}$ was observed with a higher intake of any type of high-alcoholic beverage, but in this case no conclusion can be drawn because of the high percentages of non-spirit drinkers in all study groups and the small number of drinkers at higher levels.

\section{Acknowledgements}

The authors thank the study participants for their confidence and collaboration.

This work was supported by Bracco Spa. Bracco Spa contributed to the study design and had no role in analysis or writing of this article.

The authors' contributions are as follows: M. C. was the principal investigator and contributed to the study design; 
R. A. F. contributed to the study design and wrote the manuscript; V. F. carried out data analyses and wrote the manuscript; E. G. contributed to the study design; A. D. C., S. B., D. D. C., T. L., M. D. M., O. I., R. C., M. F., F. L., V. D and G. I. contributed to subject briefings and data collection; A. R. carried out data analyses. All authors read and approved the final version of the manuscript.

The authors declare that there are no conflicts of interest.

\section{References}

1. Shaheen NJ \& Richter JE (2009) Barrett's oesophagus. Lancet 373, 850-861.

2. Schneider JL \& Corley DA (2015) A review of the epidemiology of Barrett's oesophagus and oesophageal adenocarcinoma. Best Pract Res Clin Gastroenterol 29, 29-39.

3. Koek GH, Sifrim D, Lerut T, et al. (2008) Multivariate analysis of the association of acid and duodeno-gastro-oesophageal reflux exposure with the presence of oesophagitis, the severity of oesophagitis and Barrett's oesophagus. Gut 57, 1056-1064.

4. Ronkainen J, Aro P, Storskrubb T, et al. (2005) High prevalence of gastroesophageal reflux symptoms and esophagitis with or without symptoms in the general adult Swedish population: a Kalixanda study report. Scand J Gastroenterol 40, 275-285.

5. Lee D, Lee KJ, Kim KM, et al. (2013) Prevalence of asymptomatic erosive esophagitis and factors associated with symptom presentation of erosive esophagitis. Scand J Gastroenterol 48, 906-912.

6. Bagnardi V, Rota M, Botteri E, et al. (2015) Alcohol consumption and site-specific cancer risk: a comprehensive dose-response meta-analysis. Br J Cancer 112, 580-593.

7. Thrift AP, Cook MB, Vaughan TL, et al. (2014) Alcohol and the risk of Barrett's esophagus: a pooled analysis from the International BEACON Consortium. Am J Gastroenterol 109, 1586-1594.

8. Steevens J, Schouten LJ, Driessen AL, et al. (2011) A prospective cohort study on overweight, smoking, alcohol consumption, and risk of Barrett's esophagus. Cancer Epidemiol Biomarkers Prev 20, 345-358.

9. Thrift AP, Kramer JR, Richardson PA, et al. (2014) No significant effects of smoking or alcohol consumption on risk of Barrett's esophagus. Dig Dis Sci 59, 108-116.

10. Kubo A, Levin TR, Block G, et al. (2009) Alcohol types and sociodemographic characteristics as risk factors for Barrett's esophagus. Gastroenterology 136, 806-815.

11. Xu Q, Guo W, Shi X, et al. (2015) Association between alcohol consumption and the risk of Barrett's esophagus: a metaanalysis of observational studies. Medicine (Baltimore) $\mathbf{9 4}$, e1244.

12. Wang KK \& Sampliner RE, Practice Parameters Committee of the American College of Gastroenterology (2008) Updated guidelines 2008 for the diagnosis, surveillance and therapy of Barrett's esophagus. Am J Gastroenterol 103, 788-797.

13. Sharma P, Dent J, Armstrong D, et al. (2006) The development and validation of an endoscopic grading system for Barrett's esophagus: the Prague C \& M criteria. Gastroenterology 131, 1392-1399.

14. Reid BJ, Blount PL, Feng Z, et al. (2000) Optimizing endoscopic biopsy detection of early cancers in Barrett's high-grade dysplasia. Am J Gastroenterol 95, 3089-3096.

15. Lundell LR, Dent J, Bennett JR, et al. (1999) Endoscopic assessment of oesophagitis: clinical and functional correlates and further validation of the Los Angeles classification. Gut 45, 172-180.

16. Edelstein ZR, Bronner MP, Rosen SN, et al. (2009) Risk factors for Barrett's esophagus among patients with gastroesophageal reflux disease: a community clinic-based case-control study. Am J Gastroenterol 104, 834-842.

17. Hosmer DW \& Lemeshow S (2000) Applied Logistic Regression, 2nd ed. New York, NY: Wiley \& Sons.

18. Leffondré K, Abrahamowicz M, Siemiatycki J, et al. (2002) Modeling smoking history: a comparison of different approaches. Am J Epidemiol 156, 813-823.

19. Harrell FE Jr (2015) Regression Modelling Strategies, 2nd ed. New York: Springer Verlag.

20. Ronksley PE, Brien SE, Turner BJ, et al. (2011) Association of alcohol consumption with selected cardiovascular disease outcomes: a systematic review and meta-analysis. BMJ 342, d67.

21. Eapen DJ, Manocha P, Valiani K, et al. (2011) Alcohol and the heart: an ounce of prevention. Curr Treat Options Cardiovasc Med 13, 313-325.

22. Wakabayashi I (2016) A U-shaped relationship between alcohol consumption and cardiometabolic index in middleaged men. Lipids Health Dis 15, 50.

23. Dorans KS, Mostofsky E, Levitan EB, et al. (2015) Alcohol and incident heart failure among middle-aged and elderly men: cohort of Swedish men. Circ Heart Fail 8, 422-427.

24. Krenz M \& Korthuis RJ (2012) Moderate ethanol ingestion and cardiovascular protection: from epidemiologic associations to cellular mechanisms. J Mol Cell Cardiol 52, 93-104.

25. Keshavarzian A, Rizk G, Urban G, et al. (1990) Ethanolinduced esophageal motor disorder: development of an animal model. Alcohol Clin Exp Res 14, 76-81.

26. Kawanishi M (2006) Will symptomatic gastroesophageal reflux disease develop into reflux esophagitis? J Gastroenterol $\mathbf{4 1}$, 440-443.

27. Mohammed I, Nightingale P \& Trudgill NJ (2005) Risk factors for gastrooesophageal reflux disease symptoms: a community study. Aliment Pharmacol Ther 21, 821-827.

28. Nocon M, Labenz J \& Willich SN (2006) Lifestyle factors and symptoms of gastro-oesophageal reflux - a populationbased study. Aliment Pharmacol Ther 23, 169-174.

29. Locke GR 3rd, Talley NJ, Fett SL, et al. (1999) Risk factors associated with symptoms of gastroesophageal reflux. Am J Med 106, 642-649.

30. Pandeya N, Green AC, Whiteman DC, et al. (2012) Prevalence and determinants of frequent gastroesophageal reflux symptoms in the Australian community. Dis Esophagus 25, 573-583.

31. Dore MP, Maragkoudakis E, Fraley K, et al. (2008) Diet, lifestyle and gender in gastro-esophageal reflux disease. Dig Dis Sci 53, 2027-2032.

32. Vaughan TL, Davis S, Kristal A, et al. (1995) Obesity, alcohol, and tobacco as risk factors for cancers of the esophagus and gastric cardia: adenocarcinoma versus squamous cell carcinoma. Cancer Epidemiol Biomarkers Prev 4, 85-92.

33. Kabat GC, Ng SK \& Wynder EL (1993) Tobacco, alcohol intake, and diet in relation to adenocarcinoma of the esophagus and gastric cardia. Cancer Causes Control 4 , $123-132$.

34. Garidou A, Tzonou A, Lipworth L, et al. (1996) Life-style factors and medical conditions in relation to esophageal cancer by histologic type in a low-risk population. Int J Cancer $\mathbf{6 8}$, 295-299.

35. Gammon MD, Schoenberg JB, Ahsan H, et al. (1997) Tobacco, alcohol, and socioeconomic status and adenocarcinomas of the esophagus and gastric cardia. J Natl Cancer Inst $\mathbf{8 9}$, $1277-1284$. 
36. Lagergren J, Bergstrom R, Lindgren A, et al. (2000) The role of tobacco, snuff and alcohol use in the aetiology of cancer of the oesophagus and gastric cardia. Int J Cancer $\mathbf{8 5}$, 340-346.

37. Freedman ND, Murray LJ, Kamangar F, et al. (2011) Alcohol intake and risk of oesophageal adenocarcinoma: a pooled analysis from the BEACON Consortium. Gut 60, 1029-1037.

38. Veugelers PJ, Porter GA, Guernsey DL, et al. (2006) Obesity and lifestyle risk factors for gastroesophageal reflux disease, Barrett esophagus and esophageal adenocarcinoma. Dis Esophagus 19, 321-328.

39. Wu AH, Wan P \& Bernstein L (2001) A multiethnic populationbased study of smoking, alcohol and body size and risk of adenocarcinomas of the stomach and esophagus (United States). Cancer Causes Control 12, 721-732.

40. Lindblad M, Rodriguez LA \& Lagergren J (2005) Body mass, tobacco and alcohol and risk of esophageal, gastric cardia, and gastric noncardia adenocarcinoma among men and women in a nested case-control study. Cancer Causes Control 16, 285-294.

41. Hashibe M, Boffetta P, Janout V, et al. (2007) Esophageal cancer in Central and Eastern Europe: tobacco and alcohol. Int J Cancer 120, 1518-1522.

42. Freedman ND, Abnet CC, Leitzmann MF, et al. (2007) A prospective study of tobacco, alcohol, and the risk of esophageal and gastric cancer subtypes. Am J Epidemiol 165, 1424-1433.

43. Anderson LA, Cantwell MM, Watson RG, et al. (2009) The association between alcohol and reflux esophagitis, Barrett's esophagus, and esophageal adenocarcinoma. Gastroenterology 136, 799-805.

44. Yates M, Cheong E, Luben R, et al. (2014) Body mass index, smoking, and alcohol and risks of Barrett's esophagus and esophageal adenocarcinoma: a UK prospective cohort study. Dig Dis Sci 59, 1552-1559.

45. Conio M, Filiberti R, Blanchi S, et al. (2002) Risk factors for Barrett's esophagus: a case-control study. Int J Cancer 97, $225-259$.

46. Hirota WK, Loughney TM, Lazas DJ, et al. (1999) Specialized intestinal metaplasia, dysplasia, and cancer of the esophagus and esophagogastric junction: prevalence and clinical data. Gastroenterology 116, 277-285.

47. Johansson J, Hakansson HO, Mellblom L, et al. (2007) Risk factors for Barrett's oesophagus: a population-based approach. Scand J Gastroenterol 42, 148-156.

48. Thrift AP, Pandeya N, Smith KJ, et al. (2011) Lifetime alcohol consumption and risk of Barrett's esophagus. Am J Gastroenterol 106, 1220-1230.

49. Pandeya N, Williams G, Green AC, et al. (2009) Alcohol consumption and the risks of adenocarcinoma and squamous cell carcinoma of the esophagus. Gastroenterology 136, $1215-1224$.
50. Mayne ST \& Navarro SA (2002) Diet, obesity and reflux in the etiology of adenocarcinomas of the esophagus and gastric cardia in humans. J Nutr 132, S3467-S3470.

51. Minatsuki C, Yamamichi N, Shimamoto T, et al. (2013) Background factors of reflux esophagitis and non-erosive reflux disease: a cross-sectional study of 10,837 subjects in Japan. PLOS ONE 8, e69891.

52. Avidan B, Sonnenberg A, Schnell TG, et al. (2001) Risk factors for erosive reflux esophagitis: a case-control study. Am J Gastroenterol 96, 41-46.

53. Ryan P, Hetzel DJ, Shearman DJ, et al. (1995) Risk factors for ulcerative reflux oesophagitis: a case-control study. J Gastroenterol Hepatol 10, 306-312.

54. Labenz J, Jaspersen D, Kulig M, et al. (2004) Risk factors for erosive esophagitis: a multivariate analysis based on the ProGERD study initiative. Am J Gastroenterol 99, $1652-1656$

55. Rivero-Perez MD, Muniz P \& Gonzalez-Sanjose ML (2007) Antioxidant profile of red wines evaluated by total antioxidant capacity, scavenger activity, and biomarkers of oxidative stress methodologies. I Agric Food Chem 55 , 5476-5483.

56. Kubo A \& Corley DA (2007) Meta-analysis of antioxidant intake and the risk of esophageal and gastric cardia adenocarcinoma. Am J Gastroenterol 102, 2323-2330.

57. Kubo A, Levin TR, Block G, et al. (2008) Dietary patterns and the risk of Barrett's esophagus. Am J Epidemiol 167, 839-846.

58. Bianchini F \& Vainio H (2003) Wine and resveratrol: mechanisms of cancer prevention? Eur J Cancer Prev 12, 417-425.

59. Gerhauser C (2005) Beer constituents as potential cancer chemopreventive agents. Eur J Cancer 41, 941-954.

60. Vasdev S, Gill V \& Singal PK (2006) Beneficial effect of low ethanol intake on the cardiovascular system: possible biochemical mechanisms. Vasc Health Risk Manag 2, 263-276.

61. Barefoot JC, Gronbaek M, Feaganes JR, et al. (2002) Alcoholic beverage preference, diet, and health habits in the UNC alumni heart study. Am J Clin Nutr 76, 466-472.

62. El-Serag HB, Petersen NJ, Carter J, et al. (2004) Gastroesophageal reflux among different racial groups in the United States. Gastroenterology 126, 1692-1699.

63. Nandurkar S, Locke GR III, Murray JA, et al. (2005) Rates of endoscopy and endoscopic findings among people with frequent symptoms of gastroesophageal reflux in the community. Am J Gastroenterol 100, 1459-1465.

64. El-Serag HB \& Lagergren J (2009) Alcohol drinking and the risk of Barrett's esophagus and esophageal adenocarcinoma. Gastroenterology 136, 1155-1157.

65. Shivappa N, Hébert JR, Rosato V, et al. (2016) Inflammatory potential of diet and risk of laryngeal cancer in a case-control study from Italy. Cancer Causes Control 27, 1027-1034.

66. Gronbaek M (2007) Confounders of the relation between type of alcohol and cardiovascular disease. Ann Epidemiol 17, S13-S15. 